\title{
Chest CT for detecting COVID-19: a systematic review and meta-analysis of diagnostic accuracy
}

\author{
Buyun $\mathrm{Xu}^{1} \cdot$ Yangbo Xing ${ }^{1} \cdot$ Jiahao Peng ${ }^{2} \cdot$ Zhaohai Zheng $^{3} \cdot$ Weiliang Tang $^{1} \cdot$ Yong Sun $^{1} \cdot$ Chao Xu $^{1} \cdot$ Fang Peng $^{1}$
}

Received: 14 April 2020 / Accepted: 4 May 2020 / Published online: 15 May 2020

(C) European Society of Radiology 2020

\begin{abstract}
Objective The purpose of this article was to perform a systematic review and meta-analysis regarding the diagnostic test accuracy of chest CT for detecting coronavirus disease 2019 (COVID-19).

Methods PubMed, Embase, Web of Science, and CNKI were searched up to March 12, 2020. We included studies providing information regarding diagnostic test accuracy of chest CT for COVID-19 detection. The methodological quality was assessed using the Quality Assessment of Diagnostic Accuracy Studies-2 tool. Sensitivity and specificity were pooled.

Results Sixteen studies $(n=3186$ patients) were included. The risks of bias in all studies were moderate in general. Pooled sensitivity was $92 \%(95 \% \mathrm{CI}=86-96 \%)$, and two studies reported specificity $(25 \%[95 \% \mathrm{CI}=22-30 \%]$ and $33 \%[95 \% \mathrm{CI}=23-$ 44\%], respectively). There was substantial heterogeneity according to Cochran's $Q$ test $(p<0.01)$ and Higgins $I^{2}$ heterogeneity index ( $96 \%$ for sensitivity). After dividing the studies into two groups based on the study site, we found that the sensitivity of chest CT was great in Wuhan (the most affected city by the epidemic) and the sensitivity values were very close to each other (97\%, 96\%, and 99\%, respectively). In the regions other than Wuhan, the sensitivity varied from 61 to $98 \%$.

Conclusion Chest CT offers the great sensitivity for detecting COVID-19, especially in a region with severe epidemic situation. However, the specificity is low. In the context of emergency disease control, chest CT provides a fast, convenient, and effective method to early recognize suspicious cases and might contribute to confine epidemic.

Key Points

- Chest CT has a high sensitivity for detecting COVID-19, especially in a region with severe epidemic, which is helpful to early recognize suspicious cases and might contribute to confine epidemic.
\end{abstract}

Keywords X-ray computed tomography $\cdot$ Coronaviruses $\cdot$ Pneumonias

\section{Abbreviations}

COVID-19 Coronavirus disease 19

GGO Ground-glass opacity

RT-PCR Reverse transcription polymerase chain reaction

SARS-CoV-2 Severe acute respiratory

syndrome coronavirus 2

Buyun $\mathrm{Xu}$ and Yangbo Xing contributed equally to this work.

Fang Peng

sxrmyypf@126.com

1 Department of Cardiology, Shaoxing People's Hospital (Shaoxing Hospital, Zhejiang University School of Medicine), No. 568, Zhongxing North Road, Shaoxing 312000, Zhejiang Province, People's Republic of China

\section{Introduction}

In December 2019, an outbreak of pneumonia associated with a novel coronavirus called severe acute respiratory syndrome coronavirus 2 (SARS-CoV-2) was reported in Wuhan, Hubei Province, China [1]. Thereafter, the WHO named the disease as coronavirus disease 2019 (COVID-19). With virus spreading globally, the WHO characterizes COVID-19 as a pandemic.

2 Loma Linda University School of Public Health, 24951 Circle Dr, Loma Linda, CA 92354, USA

3 Zhejiang University School of Medicine, No. 866, Yuhangtang Road, Hangzhou 310000, People's Republic of China 
The keys to control COVID-19 are early discovery, early isolation, and early treatment. At present, the real-time reverse transcription polymerase chain reaction (RT-PCR) assay remains the standard of reference, but it was reported that falsenegative RT-PCR was not rare and, in these patients, initial chest CT might present abnormal findings indicating COVID19 [2]. Moreover, shortage of laboratory test kits limited the use of RT-PCR with the spread of the epidemic, especially in regions severely affected by the epidemic. Therefore, some experts suggested that chest $\mathrm{CT}$ could be regarded as a diagnosis standard of COVID-19. The guideline of Diagnosis and Treatment of Pneumonitis Caused by 2019-nCoV (trial sixth version) published by the China government recommended chest $\mathrm{CT}$ as an effective method to screen suspicious cases [3]. The addition of chest CT for diagnosis of COVID-19 resulted in tens of thousands of clinically diagnosed cases in China which played an important role in controlling epidemic situation in China [4]. Therefore, comprehensive and timely evaluation of the effectiveness of chest CT for COVID-19 diagnosis remains urgent and mandatory. In the present study, we validated the effectiveness of chest CT for COVID-19 diagnosis through a systematical meta-analysis.

\section{Material and methods}

\section{Search strategy and eligibility criteria}

PubMed, Embase, Web of Science, and CNKI (until March 31, 2020) were searched for articles that focused on the role of chest CT in diagnosis of COVID-19; there were no language restrictions. The keywords were "COVID-19" or "SARS-CoV-2" or "novel coronavirus" or "2019 nCov." We also checked the reference lists of all key articles for any additional eligible articles. Studies were included if they met the following criteria: (1) reported the performance of chest CT in diagnosing COVID-19, (2) participants were diagnosed as COVID-19 based on the results of multiple RT-PCR, (3) studies directly or indirectly provided enough information to extract $2 \times 2$ table information of diagnostic test of chest CT for COVID-19, and (4) study sample was larger than 30 . We excluded duplicate reports, abstracts from meeting proceedings. The selection of eligible articles was performed by 2 investigators independently. Disparities between investigators were resolved by discussion between them.

\section{Data extraction and quality assessment}

The following data were extracted from each study: study site, sample size, characteristics of participants, chest CT findings, the results of multiple RT-PCR, and $2 \times 2$ table information. The methodological quality was evaluated by using the Quality Assessment of Diagnostic Accuracy Studies-2
(QUADAS-2) tool. QUADAS-2 entries include 4 domains: patient selection, index test, reference standard, and flow and timing. Definitions and judgment criteria for each domain are available in Cochrane Handbook. Data extraction and quality assessment were conducted by two independent authors. Disparities between investigators were resolved by discussion between them.

\section{Statistical analysis}

A bivariate random-effects model was used to analyze and pool diagnostic performance (sensitivity and specificity) measurements across studies. The chi square-based $Q$ test was performed to test heterogeneity among studies. And, the $I^{2}$ value was used to evaluate the percentage of interstudy variation in the total variation. When $p<0.05$ and/or $I^{2}>50 \%$, significant heterogeneity was presumed. The meta-analysis was conducted using the "midas" and "metandi" modules in Stata 12.0 software.

\section{Results}

\section{Characteristics of studies and quality assessment}

The study selection process is presented in Fig. 1. Briefly, 663 references were identified after searching databases. Eight references were removed due to duplication. After scanning the titles and abstracts, 623 records were excluded and 32 full-text articles were assessed for eligibility. Finally, 16 studies were included in our meta-analysis involving 3186 patients [5-20]. Of these patients, 2689 patients had positive RT-PCR results. Fourteen studies only enrolled patients diagnosed as COVID19 by RT-PCR [6-14, 16-20]. Therefore, we could only calculate sensitivity from the information provided in these studies. Three studies were conducted in Wuhan $[5,8,12] ; 12$ studies were conducted in the other regions, and the remaining one collected data throughout China $[6,7,9-11,14-20]$. The mean or median age of patients ranged from 37 to 62 . Seven studies reported the proportion of severe illness which ranged from 3.8 to $41 \%$ [6, 10-12, 14, 17, 19]. The characteristics of eligible studies are summarized in Table 1.

The risk of bias and applicability concerns for included studies are shown in Fig. 2. Overall, none of the studies was considered to be seriously flawed according to the QUADAS2 assessment. At least 4 of 7 items of the QUADAS-2 tool were met in all studies. All studies were considered to have a low risk of bias in the patient selection and reference standard domain. Only two studies reported that the radiologists reading the chest $\mathrm{CT}$ images were blinded to the results of RT-PCR which was classified as low risk of bias in the index test domain $[5,20]$. The remaining studies were considered to have an unclear risk of bias regarding the index test domain. 


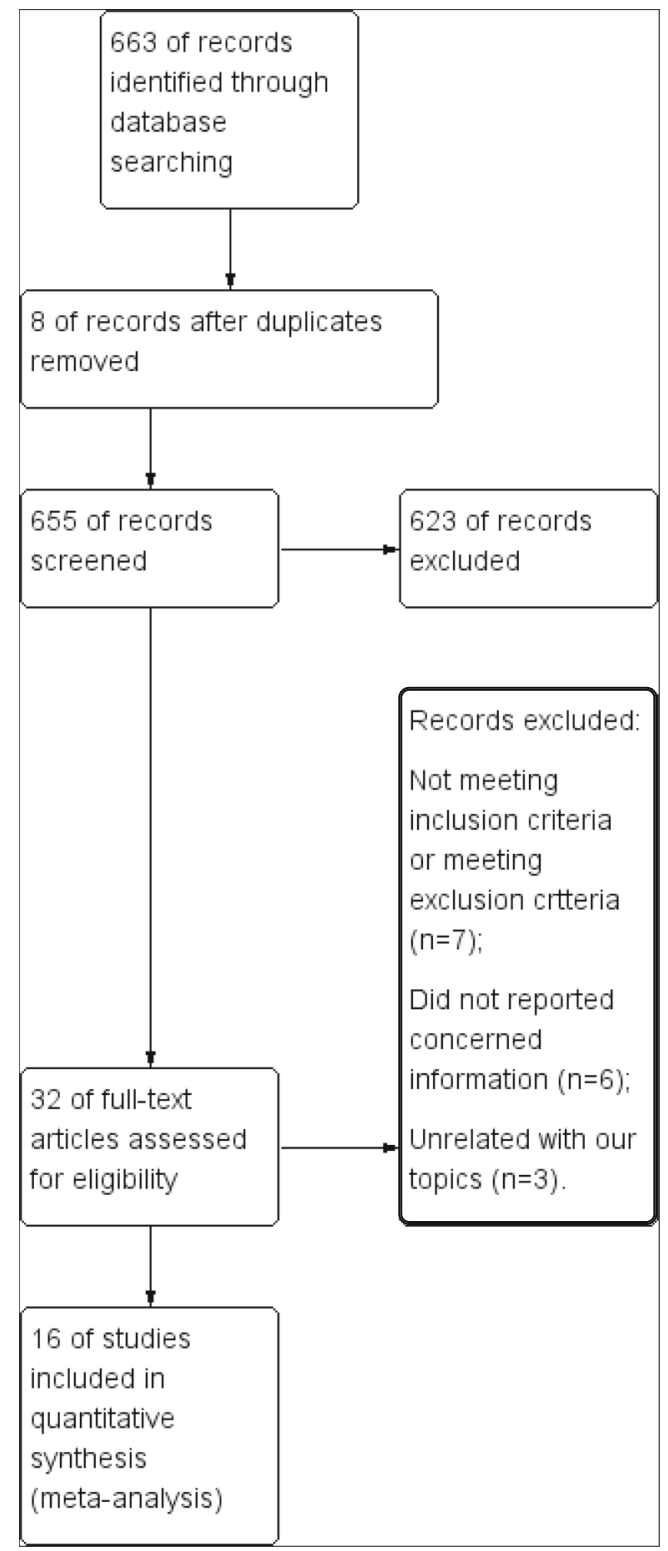

Fig. 1 Flow diagram showing the study selection process for metaanalysis

With regard to the flow and timing domain, we noted that 11 studies had an ambiguous risk of bias due to the absence of mean interval data between chest CT and the RT-PCR assay $[6,7,10-14]$. And, the remaining studies reported a reasonable interval between chest CT and the RT-PCR assay (13 days) $[5,8,9,15,19]$.

\section{Diagnostic performance of chest CT for diagnosing COVID-19}

Sensitivity was available in all studies ranging from 0.61 to 0.99 [5-20]. However, only two studies reported the specificity of chest CT for diagnosing COVID-19 about 25\% (95\% $\mathrm{CI}=21-30 \%)$ and $33 \%(95 \% \mathrm{CI}=23-44 \%)$, respectively (due to the significant heterogeneity and small number of studies, it was inappropriate to pool the data). We pooled the sensitivity values $(92 \%, 95 \% \mathrm{CI}=86-96 \%)$ (Fig. 3). Cochran's $Q$ test revealed a significant heterogeneity $(Q=$ $\left.419, p<0.01 ; I^{2}=96.4\right)$. Given that the severity of illness, experience of radiologists, and severity of epidemic might contribute to the heterogeneity, we classified the studies into two categories according to whether or not the study site was located in Wuhan where the epidemic was the most severe, patients suffered more severe illness and the radiologists might have more experience. Three studies were conducted in Wuhan which reported sensitivity values about $97 \%$ (95\% $\mathrm{CI}=95-98 \%)[5], 96 \%(0.87-100 \%)[8]$, and $99 \%(95 \%$ $\mathrm{CI}=96-100 \%)[12]$, respectively. Due to the small number of enrolled studies, it was inappropriate to pool sensitivity among these three studies, but the sensitivity values were very close to each other. After excluding these three studies, we pooled the sensitivity values of the remaining studies. Nevertheless, the heterogeneity was still so significant that it was inappropriate to pool the sensitivity $(Q=212, p<0.01$; $I^{2}=94.5$ ). The sensitivity value of the individual study ranged from 0.61 to 0.98 and is summarized in Fig. 3.

Due to the specificity only reported in two studies [5, 15], it was inappropriate to plot hierarchical summary receiver operating characteristic (HSROC) curves.

In addition to the significant heterogeneity of the value of chest $\mathrm{CT}$ in diagnosing COVID-19, the CT findings in COVID-19 were also varied across enrolled studies. The characteristics of chest CT findings in COVID-19 patients with abnormal CT images are summarized in Table 2. Although the reported proportion might vary across different studies, ground-glass opacity (GGO) and consolidative opacities were some of the most common CT findings, with reporting rates about $49-94 \%$ and $11-73 \%$, respectively. Besides, interlobular septal thickening, pleural thickening, and bronchiectasis were also reported with various rates across the studies. With regard to lesion distribution, almost all studies reported that involvement of multiple lobes of both lungs was most common (65-97\%). Peripheral zone and lower lobes were more predisposed to be affected.

In the present review, we also summarized the performance of chest CT in patients with initial false-negative RT-PCR (Table 2). Three studies reported related information $[5,9$, 16]. After combining data among these studies, 36 patients had initial false-negative RT-PCR, but $31 / 36$ patients had positive initial chest $\mathrm{CT}$.

\section{Discussion}

The main results of this meta-analysis are as follows: (1) the sensitivity of chest CT for COVID-19 was great in Wuhan but varied among other regions, (2) few studies reported the 
Table 1 Characteristics of enrolled studies

\begin{tabular}{|c|c|c|c|c|c|c|}
\hline Author & Sample size & Site & Gender (male, \%) & Age & Severity (severe, \%) & $\begin{array}{l}\text { Median interval between } \\
\text { CT and RT-PCR (days) }\end{array}$ \\
\hline $\mathrm{Ai}$ et al [5] & 1014 & Wuhan, China & 46 & Mean age, 51 & NA & 1 \\
\hline Guan et al [6] & $1099^{\mathrm{a}}$ & Throughout China & 58.1 & Median age, 47 & 15.7 & NA \\
\hline Xu et al [7] & 62 & Not Wuhan, China & 56 & Median age, 47 & NA & NA \\
\hline $\mathrm{Li}$ and $\mathrm{Xia}[8]$ & 51 & Wuhan, China & 55 & Mean age, 58 & NA & 3 \\
\hline Fang et al [9] & 51 & Not Wuhan, China & 57 & Median age, 45 & NA & 3 \\
\hline Yang et al [10] & 149 & Not Wuhan, China & 54 & Mean age, 45 & 8.7 & NA \\
\hline Wu et al [11] & 80 & Not Wuhan, China & 51 & Mean age, 46 & 3.8 & NA \\
\hline Zhang et al [12] & $140^{\mathrm{a}}$ & Wuhan, China & 49 & Median age, 57 & 41 & NA \\
\hline Xu et al [13] & 90 & Not Wuhan, China & 43 & Mean age, 50 & NA & NA \\
\hline Xu et al [14] & 50 & Not Wuhan, China & 58 & Median age, 45 & 26.0 & NA \\
\hline Zhu et al [15] & 116 & Not Wuhan, China & 46 & Median age, 40 & NA & $<1$ \\
\hline Long et al [16] & 36 & Not Wuhan, China & 56 & Mean age, 45 & NA & NA \\
\hline Li et al [17] & 78 & Not Wuhan, China & 49 & Mean age, 45 & 10.3 & NA \\
\hline Wang et al [18] & 114 & Not Wuhan, China & 51 & Mean age, 53 & NA & NA \\
\hline Liu et al [19] & 73 & Not Wuhan, China & 56 & Mean age, 37 & 33 & $<2$ in $88 \%$ of patients \\
\hline Inui et al [20] & 112 & Japan & 53 & Mean age, 62 & NA & NA \\
\hline
\end{tabular}

$N A$ not available

${ }^{a}$ Not all patients received chest $\mathrm{CT}$

specificity of chest CT which was about 25-33\%, (3) typical chest CT findings of COVID-19 were GGO and consolidative opacities which involved multiple lobes of both lungs, and (4) chest CT had a high sensitivity in patients with initial falsenegative RT-PCR.

Chest CT plays an important role in detection of COVID19 , especially in the initial and peak periods of epidemic, in China. Although the RT-PCR assay remains the standard of reference, it has been reported that false-negative result after the initial test was not rare and shortage of laboratory kit in the early stage of the outbreak restricted the early detection of COVID-19. As our findings, chest CT had great sensitivity for early detection of COVID-19, especially in regions more affected by epidemic such as Wuhan. Therefore, a clinical diagnosis criterion based on typical CT imaging features was temporarily adopted in the guideline of diagnosis and treatment, which was only applicable in Hubei Province, China [3]. This move allowed to early detect a large number of clinical diagnoses of COVID-19 under the background of shortage of RT-PCR assay which contributed to effective control of epidemic situation in China. Nevertheless, as mentioned above, among regions other than Wuhan, the reported sensitivity of chest CT varied and was generally lower than that in Wuhan. Several reasons might underlie this phenomenon, such as heterogeneity of experience of radiologists, severity of illness, and epidemic. However, unfortunately, most included studies did not provide related data to further confirm this hypothesis. Wu et al [11] reported a relatively low sensitivity of chest CT (69\%), and in their study, the proportion of severe patients was only about $3.8 \%$ far less than the average

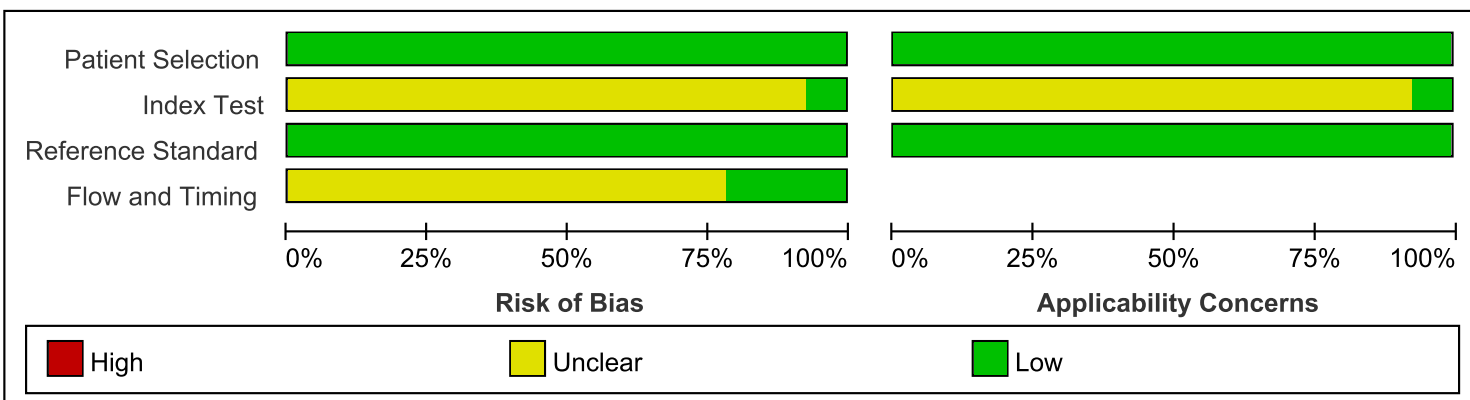

Fig. 2 Grouped bar charts of risk of bias (left) and concerns for applicability (right) of 10 included studies using the Quality Assessment of Diagnostic Accuracy Studies-2 (QUADAS-2) tool 
level of China which was about $15 \%$ [6]. The reported sensitivity of chest CT by Inui et al [20] was particularly low among enrolled studies, about $61 \%$. Although the authors did not report the severity of enrolled patients directly, $73 \%$ of patients were asymptomatic and the remaining patients only had mild symptoms [20]. Li et al [17] and Liu et al [19] analyzed the CT results according to the severity of illness and found that almost all mild patients had normal CT images. In addition to severity of illness, Bai et al [21] investigated the performance of radiologists in reading chest $\mathrm{CT}$ images of COVID-19 which found the experience of radiologists had a great impact on the diagnosis accuracy of chest CT. Overall, chest CT has a great sensitivity for detecting COVID-19, especially in regions with severe epidemic situation, and is helpful to early detect suspicious cases, which is vital to control epidemic.

In contrast to the great sensitivity of chest $\mathrm{CT}$, the specificity was relatively low with reporting about $25-33 \%$. According to our review, the typical chest CT findings of COVID-19 included GGOs, consolidations, and interlobular thickening, which were usually multifocal and involved bilateral lungs, which was consistent with the results by Salehi et al [22]. In mild patients or early period of COVID-19, chest CT could be negative or pure GGO lesions. The CT imaging features might overlap between COVID-19 and other viral pneumonias, which could reduce the specificity of chest COVID-19. Due to the low specificity of chest CT which might overwhelm available recourses, especially during an influenza epidemic, the American College of Radiology discourages systemic use for diagnosing COVID-19 [23]. Nevertheless, with further investigation and more experienced radiologist, more and more features have been found to be helpful in distinguishing COVID-19 from other pneumonias, such as multifocal or bilateral involvement. So, the specificity of chest $\mathrm{CT}$ might improve in the future. In addition, considering the rapidly spreading epidemic of COVID-19, it was a priority to identify any suspicious case in order to isolate the patients and avoid cross infection. Therefore, in the context of emergency disease control, sensitivity was more important than specificity. On the other hand, as mentioned above, although RT-PCR was still regarded as standard reference, false-negative results were not rare. In our review, more than $5 \%$ of patients had initial false-negative RT-PCR results and turned positive after multiple tests. However, $86 \%$ of these patients presented positive chest $\mathrm{CT}$ before the initial negative RT-PCR results. And, more and more cases with initial false-negative RT-PCR but initial positive chest CT have been reported [24]. Last but not the least, chest CT alone could not diagnose COVID-19. According to the guideline of Diagnosis and Treatment of Pneumonitis Caused by 2019nCoV (trial sixth version) published by the China government, typical chest $\mathrm{CT}$ findings could be a diagnostic criterion to screen suspected cases when combined with epidemiology history, clinical manifestations, and laboratory results [3]. Given the concerns over false-negative result and limited availability of RT-PCR and a continuing increase in global cases, the British Society of Thoracic Imaging also underscores the importance of radiographic assessment, especially when there is diagnostic uncertainty [25].

In addition to detection, chest $\mathrm{CT}$ also plays an important role in the management of COVID-19. As other pneumonias, the severity of COVID-19 is also positively related to chest CT findings. Intensive care unit (ICU) patients on admission often presented with bilateral multiple lobular and subsegmental consolidations, while non-ICU patients presented with bilateral GGOs and subsegmental consolidation [26]. Moreover, chest $\mathrm{CT}$ is helpful to monitor disease progression of COVID-19. Pan et al [27] investigated 21 confirmed patients and summarized four stages of COVID-19: early, progressive, peak, and absorption. Growth of GGOs and
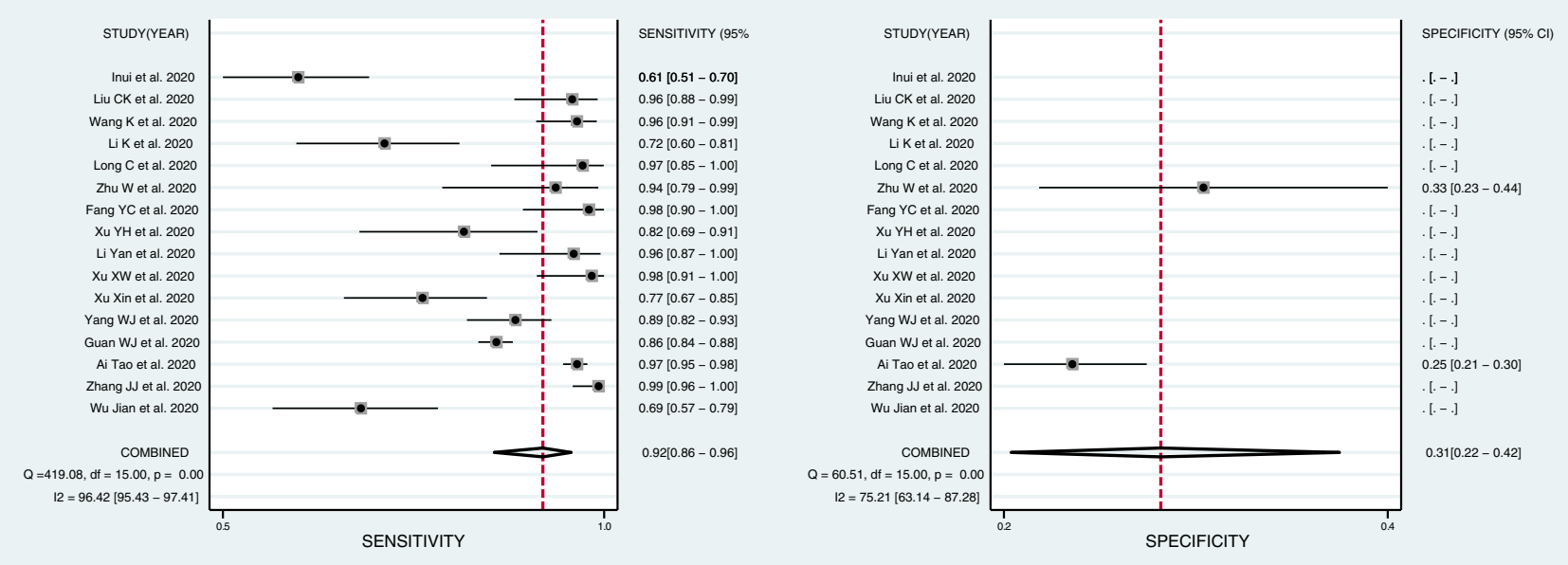

Fig. 3 Coupled forest plots of pooled sensitivity and specificity. Numbers are pooled estimates (dots within squares) with 95\% CIs (horizontal lines). Corresponding heterogeneity statistics are provided at the bottom 


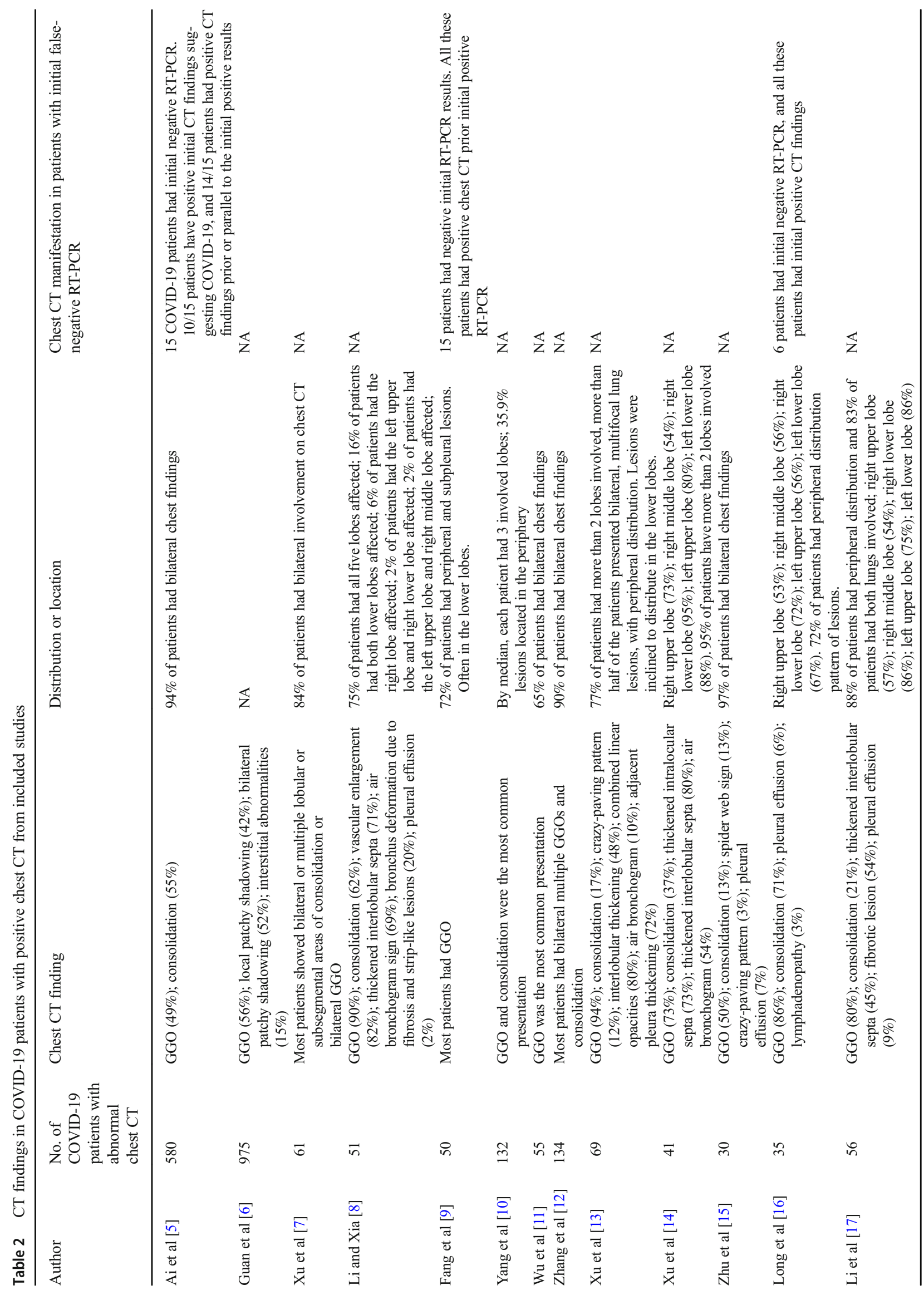


expansion of consolidation are indicators of disease progression and, otherwise, might indicate the improvement [28-31].

In conclusion, on the basis of limited and heterogeneous data, chest CT offers the great sensitivity for detecting COVID-19, especially in a region with severe epidemic situation. The specificity is low. In the context of emergency disease control, chest $\mathrm{CT}$ provides a fast, convenient, and effective method to early recognize suspicious cases and contributes to reduction of cross infection.

Funding Information The authors state that this work has not received any funding.

\section{Compliance with ethical standards}

Guarantor The scientific guarantor of this publication is Fang Peng.

Conflict of interest The authors of this manuscript declare no relationships with any companies, whose products or services may be related to the subject matter of the article.

Statistics and biometry One of the authors has significant statistical expertise.

Informed consent Written informed consent was not required for this study because this is a meta-analysis.

Ethical approval Institutional review board approval was not required because this is a meta-analysis.

Methodology

- Meta-analysis

\section{References}

1. Wang DW, Hu B, Hu C et al (2020) Clinical characteristics of 138 hospitalized patients with 2019 novel coronavirus-infected pneumonia in Wuhan, China. JAMA. https://doi.org/10.1001/jama. 2020.1585

2. Chan JF, Yuan SF, Kok KH et al (2020) A familial cluster of pneumonia associated with the 2019 novel coronavirus indicating person-toperson transmission: a study of a family cluster. Lancet. https:/doi.org/ 10.1016/S0140-6736(20)30154-9

3. General Office of National Health Committee. Notice on the issuance of a program for the diagnosis and treatment of novel coronavirus (2019-nCoV) infected pneumonia (trial sixth edition). Available via http://www.nhc.gov.cn/yzygj/s7653p/202002/ 8334a8326dd94d329df351d7da8aefc2. Accessed 18 Feb 2020

4. Zu ZY, Jiang MD, Xu PP et al (2020) Coronavirus disease 2019 (COVID-19): a perspective from China. Radiology. https://doi.org/ 10.1148/radiol.2020200490

5. Ai T, Yang ZL, Hou HY et al (2020) Correlation of chest CT and RT-PCR testing in coronavirus disease 2019 (COVID-19) in China: a report of 1014 cases. Radiology. https://doi.org/10.1148/radiol. 2020200642

6. Guan WJ, Ni ZY, Hu Y et al (2020) Clinical characteristics of coronavirus disease 2019 in China. N Engl J Med. https://doi.org/ 10.1056/NEJMoa2002032 
7. Xu XW, Wu XX, Jiang XG et al (2020) Clinical findings in a group of patients infected with the 2019 novel coronavirus (SARS-Cov-2) outside of Wuhan, China: retrospective case series. BMJ. https:// doi.org/10.1136/bmj.m606

8. Li Y, Xia LM (2020) Coronavirus disease 2019 (COVID-19): role of chest CT in diagnosis and management. AJR Am J Roentgenol. https://doi.org/10.2214/AJR.20.22954

9. Fang YC, Zhang HQ, Xie JC et al (2020) Sensitivity of chest CT for COVID-19: comparison to RT-PCR. Radiology. https:// doi.org/10.1148/radiol.2020200432

10. Yang WJ, Cao QQ, Qin L et al (2020) Clinical characteristics and imaging manifestations of the 2019 novel coronavirus disease (COVID-19): a multi-center study in Wenzhou city, Zhejiang, China. J Infect. https://doi.org/10.1016/j.jinf.2020.02.016

11. Wu J, Liu J, Zhao XG et al (2020) Clinical characteristics of imported cases of COVID-19 in Jiangsu Province: a multicenter descriptive study. Clin Infect Dis. https://doi.org/10.1093/cid/ ciaa199

12. Zhang JJ, Dong X, Cao YY et al (2020) Clinical characteristics of 140 patients infected with SARS-CoV-2 in Wuhan, China. Allergy. https://doi.org/10.1111/all.14238

13. $\mathrm{Xu} \mathrm{X,} \mathrm{Yu} \mathrm{CC,} \mathrm{Qu} \mathrm{J} \mathrm{et} \mathrm{al} \mathrm{(2020)} \mathrm{Imaging} \mathrm{and} \mathrm{clinical} \mathrm{features} \mathrm{of}$ patients with 2019 novel coronavirus SARS-CoV-2. Eur J Nucl Med Mol Imaging. https://doi.org/10.1007/s00259-020-04735-9

14. Xu YH, Dong JH, An WM et al (2020) Clinical and computed tomographic imaging features of novel coronavirus pneumonia caused by SARS-CoV-2. J Infect. https://doi.org/10.1016/j.jinf. 2020.02.017

15. Zhu W, Xie K, Lu H, Xu L, Zhou S, Fang S (2020) Initial clinical features of suspected coronavirus disease 2019 in two emergency departments outside of Hubei, China. J Med Virol. https://doi.org/ 10.1002/jmv. 25763

16. Long $\mathrm{C}, \mathrm{Xu} \mathrm{H}$, Shen $\mathrm{Q}$ et al (2020) Diagnosis of the coronavirus disease (COVID-19): rRT-PCR or CT? Eur J Radiol. https://doi.org/ 10.1016/j.ejrad.2020.108961

17. Li K, Fang Y, Li W et al (2020) CT image visual quantitative evaluation and clinical classification of coronavirus disease (COVID-19). Eur Radiol. https://doi.org/10.1007/s00330-02006817-6

18. Wang K, Kang S, Tian R, Zhang X, Zhang X, Wang Y (2020) Imaging manifestations and diagnostic value of chest $\mathrm{CT}$ of coronavirus disease 2019 (COVID-19) in the Xiaogan area. Clin Radiol. https://doi.org/10.1016/j.crad.2020.03.004

19. Liu KC, Xu P, Lv WF et al (2020) CT manifestations of coronavirus disease-2019: a retrospective analysis of 73 cases by disease severity. Eur J Radiol. https://doi.org/10.1016/j.ejrad.2020.108941

20. Inui S, Fujikawa A, Jitsu M et al (2020) Chest CT findings in cases from the cruise ship "Diamond Princess" with coronavirus disease
2019 (COVID-19). Radiology: Cardiothoracic Imaging. https://doi. org/10.1148/ryct.2020200110

21. Bai HX, Hsieh B, Xiong Z et al (2020) Performance of radiologists in differentiating COVID-19 from viral pneumonia on chest CT. Radiology. https://doi.org/10.1148/radiol.2020200823

22. Salehi S, Abedi A, Balakrishnan S, Gholamrezanezhad A (2020) Coronavirus disease 2019 (COVID-19): a systematic review of imaging findings in 919 patients. AJR Am J Roentgenol. https://doi. org/10.2214/AJR.20.23034

23. American College of Radiology. ACR recommendations for the use of chest radiography and computed tomography $(\mathrm{CT})$ for suspected COVID-19 infection. Available via https://www.acr.org/Advocacyand-Economics/ACR-PositionStatements/Recommendations-forChestRadiography-and-CT-for-Suspected-COVID19-Infection. Accessed 22 March 2020

24. Xie X, Zhong Z, Zhao W, Zheng C, Wang F, Liu J (2020) Chest CT for typical 2019-nCoV pneumonia: relationship to negative RTPCR testing. Radiology. https://doi.org/10.1148/radiol.2020200343

25. British Society of Thoracic Imaging. Radiology decision tool for suspected COVID-19. Available via https://www.bsti.org.uk/ media/resources/files/NHSE_BSTI_APPROVED_Radiology_on_ CoVid19_v6_ucQ1tNv. Accessed 16 March 2020

26. Huang CL, Wang YM, Li XW et al (2020) Clinical features of patients infected with 2019 novel coronavirus in Wuhan, China. Lancet. https://doi.org/10.1016/S0140-6736(20)30183-5

27. Pan F, Ye TH, Sun P et al (2020) Time course of lung changes on chest CT during recovery from 2019 novel coronavirus (COVID19) pneumonia. Radiology. https://doi.org/10.1148/radiol. 2020200370

28. Song FX, Shi NN, Shan F et al (2020) Emerging 2019 novel coronavirus (2019-nCoV) pneumonia. Radiology. https://doi.org/10. 1148/radiol.2020200274

29. Shi H, Han X, Zheng C (2020) Evolution of CT manifestations in a patient recovered from 2019 novel coronavirus (2019-nCoV) pneumonia in Wuhan, China. Radiology. https://doi.org/10.1148/radiol. 2020200269

30. Duan YN, Qin J (2020) Pre- and posttreatment chest CT findings: 2019 novel coronavirus (2019-nCoV) pneumonia. Radiology. https://doi.org/10.1148/radiol.2020200323

31. Wu Y, Xie Y, Wang X (2020) Longitudinal CT findings in COVID19 pneumonia: case presenting organizing pneumonia pattern. Radiology: Cardiothoracic Imaging. https://oi.org/10.1148/ryct. 2020200031

Publisher's note Springer Nature remains neutral with regard to jurisdictional claims in published maps and institutional affiliations. 